\title{
Complex Langevin calculations in QCD at finite density
}

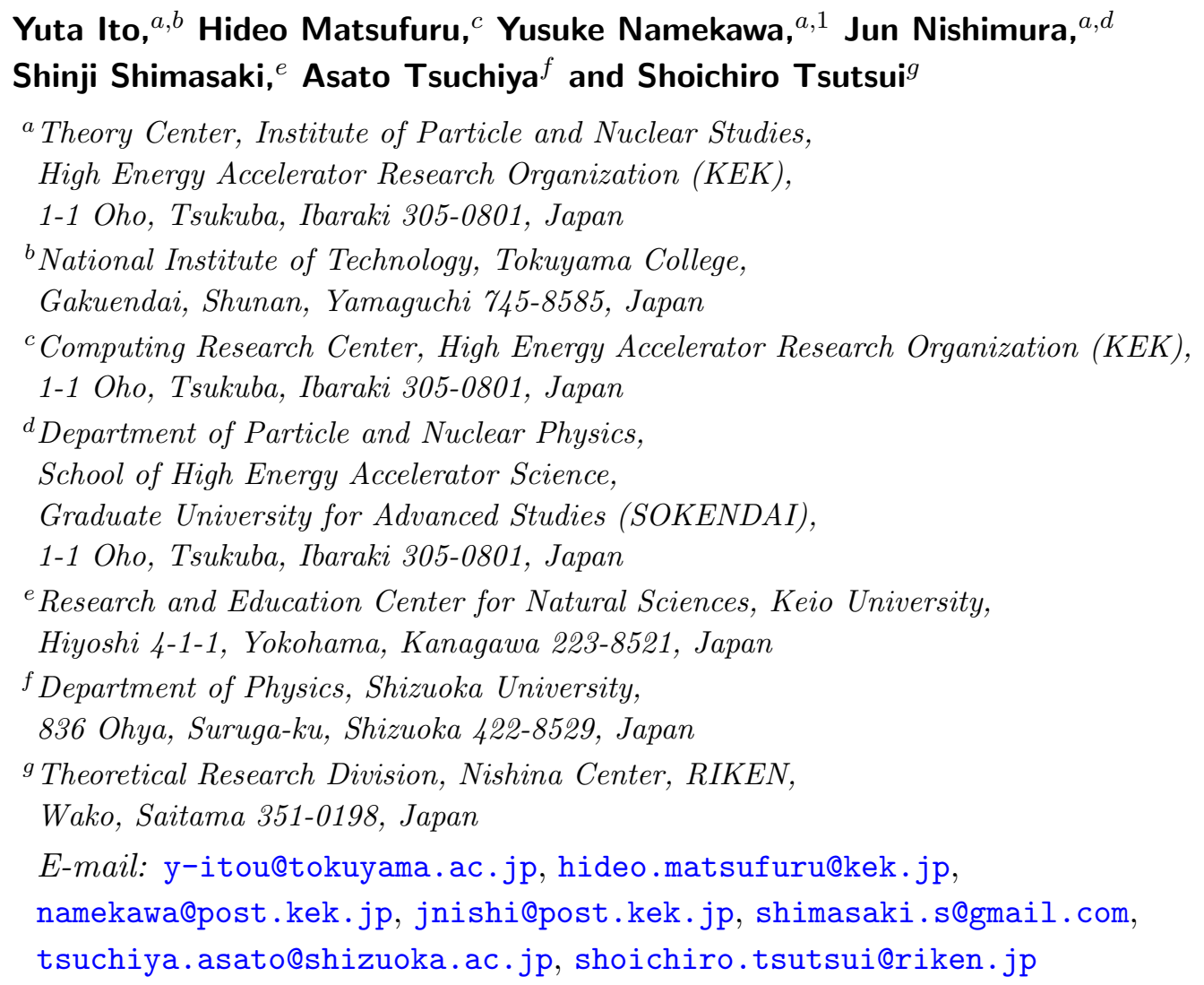

ABSTRACT: We demonstrate that the complex Langevin method (CLM) enables calculations in QCD at finite density in a parameter regime in which conventional methods, such as the density of states method and the Taylor expansion method, are not applicable due to the severe sign problem. Here we use the plaquette gauge action with $\beta=5.7$ and four-flavor staggered fermions with degenerate quark mass $m a=0.01$ and nonzero quark chemical potential $\mu$. We confirm that a sufficient condition for correct convergence is satisfied for $\mu / T=5.2-7.2$ on a $8^{3} \times 16$ lattice and $\mu / T=1.6-9.6$ on a $16^{3} \times 32$ lattice.

\footnotetext{
${ }^{1}$ Present address is Yukawa Institute for Theoretical Physics, Kyoto University, Kitashirakawa Oiwakecho, Sakyo-ku, Kyoto 606-8502 Japan.
} 
In particular, the expectation value of the quark number is found to have a plateau with respect to $\mu$ with the height of 24 for both lattices. This plateau can be understood from the Fermi distribution of quarks, and its height coincides with the degrees of freedom of a single quark with zero momentum, which is 3 (color) $\times 4$ (flavor) $\times 2$ (spin) $=24$. Our results may be viewed as the first step towards the formation of the Fermi sphere, which plays a crucial role in color superconductivity conjectured from effective theories.

KEYwords: Lattice QCD, Phase Diagram of QCD

ARXIV EPRINT: 2007.08778 


\section{Contents}

1 Introduction 1

2 CLM for QCD at finite density 3

3 Results 5

3.1 Validity of the CLM 5

$\begin{array}{lll}3.2 & \text { Physical observables } & 7\end{array}$

$\begin{array}{lll}4 & \text { Summary and discussions } & 10\end{array}$

\section{Introduction}

QCD at finite temperature and density attracts a lot of interest due to its rich phase diagram predicted by effective theories. Heavy-ion collision experiments are being performed to elucidate the phase structure, whereas the observation of gravitational waves is expected to provide significant information on the equation of state of neutron stars reflecting the phase structure. In parallel, many efforts have been made toward theoretical understanding of QCD at finite temperature and density. The difficulty in theoretical analyses, however, is that nonperturbative calculations based on lattice QCD suffer from the sign problem at finite density. This problem occurs because of the complex fermion determinant, which prevents us from applying standard Monte Carlo methods based on important sampling by identifying the Boltzmann weight as the probability distribution.

Here we focus on the complex Langevin method (CLM) [1, 2], which has recently proven a promising method for solving the sign problem. It is a complex extension of the stochastic quantization based on the Langevin equation, where dynamical variables are complexified and physical quantities as well as the drift term are extended holomorphically. An expectation value can be obtained as an average over the fictitious time evolution by the complex Langevin equation after thermalization. See ref. [3] for a summary of the recent progress concerning this method and other methods for solving the sign problem. In particular, the CLM has been tested extensively in lattice QCD at finite density [4-19].

An important issue in the CLM is that physical observables converge to wrong results depending on the parameters, the model, or even on how the method is implemented. It was not until recently that the causes of this incorrect convergence were clarified [20-27]. There are actually two kinds of causes; one is the excursion problem [20] and the other is the singular drift problem $[22,28]$. In the case of finite density QCD, the excursion problem occurs when the link variables have long excursions away from the SU(3) group manifold. This problem can be circumvented by adding the gauge cooling procedure after each Langevin update as proposed in refs. [29, 30] and justified in refs. [23, 24]. The singular 
drift problem occurs, on the other hand, when the fermion matrix has eigenvalues close to zero since the drift term involves the inverse of the fermion matrix.

Both these problems can be detected by just probing the magnitude of the drift term [24], which is calculated anyway for the Langevin evolution. If the histogram of the drift falls off exponentially or faster, one can trust the results, as has been shown from a refined argument for justifying the CLM [24] based on the discrete Langevin-time formulation. The validity of this criterion has been confirmed explicitly in simple one-variable models [24] as well as in exactly solvable models involving infinite degrees of freedom such as chiral Random Matrix Theory [27].

An alternative criterion for correct convergence has been discussed from the viewpoint of the boundary terms [31,32], which appear in the original argument [20,21] for justifying the CLM based on the continuous Langevin-time formulation. Note, however, that the limit of taking the Langevin-time stepsize to zero and the notion of time-evolved observables, which are crucial in the original argument, can be subtle when the drift histogram does not fall off fast enough [24]. These subtleties are taken into account in the refined argument, which led to the above criterion for the validity of the CLM.

In applications to QCD at finite density, it is therefore of primary importance to determine the parameter region in which the CLM gives correct results. We address this issue using staggered fermions corresponding to QCD with four-flavor quarks, which is known to have a first order deconfining phase transition at finite temperature $T$ for zero quark chemical potential $\mu=0$ [33]. The phase structure of four-flavor QCD on the $T-\mu$ plane has been investigated by various methods including the CLM using either staggered fermions $[4,6,11-14,18,34-41]$ or Wilson fermions [19, 42-46]. The strong coupling expansion was also applied as reviewed in refs. [47, 48].

In our calculations, we use Wilson's plaquette action with $\beta=5.7$ on $8^{3} \times 16$ and $16^{3} \times 32$ lattices. The quark mass for the four-flavor staggered fermions is set to $m a=0.01$. The criterion for correct convergence of the CLM is found to be satisfied for $\mu / T=5.2-7.2$ on a $8^{3} \times 16$ lattice and $\mu / T=1.6-9.6$ on a $16^{3} \times 32$ lattice, where conventional methods, such as the density of states method and the Taylor expansion method, are not applicable due to the severe sign problem.

In particular, our calculations reveal, for the first time, a plateau behavior of the quark number with respect to the quark chemical potential with the height of 24 , which can be understood from the Fermi distribution of quarks. It actually coincides with the number of degrees of freedom for a single quark with zero momentum, which is 3 (the number of colors $) \times 4$ (the number of flavors) $\times 2$ (the number of spin degrees of freedom). This can be regarded as the first step towards the formation of the Fermi surface, which plays a crucial role in color superconductivity. We also investigate other observables such as the Polyakov loop and the chiral condensate. Part of our results have been presented in proceedings articles [12-14, 18].

This paper is organized as follows. In section 2 we explain how we apply the CLM to lattice QCD at finite density. In section 3 we discuss the validity of the CLM in our simulation setup and present our results with emphasis on their physical interpretation. Section 4 is devoted to a summary and discussions. 


\section{CLM for QCD at finite density}

The partition function of QCD on a four-dimensional lattice is given by

$$
Z=\int \prod_{x, \mu} d U_{x, \mu} \operatorname{det} M[U] e^{-S_{g}[U]},
$$

where $U_{x, \mu} \in \mathrm{SU}(3)$ is a link variable in the $\mu(=1,2,3,4)$ direction with $x=\left(x_{1}, x_{2}, x_{3}, x_{4}\right)$ representing a lattice site. For the gauge action $S_{g}[U]$, we use the plaquette action

$$
S_{g}[U]=-\frac{\beta}{6} \sum_{x} \sum_{\mu \neq \nu} \operatorname{tr}\left(U_{x, \mu} U_{x+\hat{\mu}, \nu} U_{x+\hat{\nu}, \mu}^{-1} U_{x, \nu}^{-1}\right)
$$

where $\beta=6 / g^{2}$ with the gauge coupling $g$ and $\hat{\mu}$ represents the unit vector in the $\mu$ direction. In this paper, we consider the four-flavor staggered fermions with degenerate quark mass $m$ and quark chemical potential $\mu$, which corresponds to the fermion matrix

$$
M_{x y}[U]=\tilde{m} \delta_{x y}+\sum_{\mu=1}^{4} \frac{\eta_{\mu}(x)}{2}\left(e^{\tilde{\mu} \delta_{\mu 4}} U_{x, \mu} \delta_{x+\hat{\mu}, y}-e^{-\tilde{\mu} \delta_{\mu 4}} U_{x-\hat{\mu}, \mu}^{-1} \delta_{x-\hat{\mu}, y}\right),
$$

with $\eta_{\mu}(x) \equiv(-1)^{x_{1}+\cdots+x_{\mu-1}}$ being a site-dependent sign factor. In (2.3) we have defined dimensionless parameters $\tilde{m}=m a$ and $\tilde{\mu}=\mu a$, where $a$ represents the lattice spacing. The determinant $\operatorname{det} M[U]$ in $(2.1)$ becomes complex for $\tilde{\mu} \neq 0$, which causes the sign problem. Periodic boundary conditions are assumed except that we impose anti-periodic boundary conditions on the fermion fields in the temporal direction so that the temporal extent of the lattice represents the inverse temperature $T^{-1}$.

We apply the CLM to this system. In this method, the link variables $U_{x, \mu} \in \mathrm{SU}(3)$ are complexified to $\mathcal{U}_{x, \mu} \in \mathrm{SL}(3, \mathrm{C})$, and we consider a fictitious time evolution governed by

$$
\mathcal{U}_{x, \mu}(t+\varepsilon)=\exp \left\{i\left(-\varepsilon v_{x, \mu}[\mathcal{U}(t)]+\sqrt{\varepsilon} \eta_{x, \mu}(t)\right)\right\} \mathcal{U}_{x, \mu}(t)
$$

which is the discrete version of the complex Langevin equation with the stepsize $\varepsilon$. Here $\eta_{x, \mu}(t)$ is the noise term, which is a traceless $3 \times 3$ Hermitian matrix obeying the Gaussian distribution $\exp \left[-\frac{1}{4} \operatorname{tr} \eta_{x, \mu}^{2}(t)\right]$ so that

$$
\left\langle\eta_{x, \mu}^{i j}(s) \eta_{y, \nu}^{k l}(t)\right\rangle_{\eta}=2 \delta_{x y} \delta_{\mu \nu} \delta_{s t}\left(\delta_{i l} \delta_{j k}-\frac{1}{3} \delta_{i j} \delta_{k l}\right),
$$

where the symbol $\langle\cdot\rangle_{\eta}$ represents the expectation value with respect to the Gaussian noise $\eta$. The $v_{x, \mu}$ in eq. (2.4) is the drift term, which is defined by holomorphically extending

$$
v_{x, \mu}[U]=\left.\sum_{a} \lambda_{a} \frac{\partial}{\partial \alpha} S\left[e^{i \alpha \lambda_{a}} U_{x, \mu}\right]\right|_{\alpha=0}
$$

defined for unitary configurations $U$, where $S[U]=S_{g}[U]-\ln \operatorname{det} M[U]$ and $\lambda_{a}(a=$ $1, \ldots, 8)$ are the $\mathrm{SU}(3)$ generators normalized by $\operatorname{tr}\left(\lambda_{a} \lambda_{b}\right)=\delta_{a b}$. Note that the drift term 
is not Hermitian for $\tilde{\mu} \neq 0$ even for unitary link variables, which makes the time-evolved link variables inevitably non-unitary.

In order to calculate the drift term

$$
v_{x, \mu}^{(\mathrm{f})}=-\sum_{a} \lambda_{a} \operatorname{tr}\left(\left.M^{-1} \frac{\partial}{\partial \alpha} M\left[e^{i \alpha \lambda_{a}} U_{x, \mu}\right]\right|_{\alpha=0}\right)
$$

obtained from the fermion determinant, we use the standard noisy estimator. See appendix A of ref. [11] for the details.

The expectation value of an observable $\mathcal{O}(\mathcal{U})$ is calculated as

$$
\overline{\mathcal{O}}=\frac{1}{\tau} \int_{t_{0}}^{t_{0}+\tau} d t \mathcal{O}(\mathcal{U}(t))
$$

using the notation of continuum Langevin time $t$ for simplicity. Here $t_{0}$ represents the time needed for thermalization and $\tau$ represents the total Langevin time for taking the average, which should be large enough to achieve good statistics. The CLM is justified if the expectation value $\overline{\mathcal{O}}$ obtained by the CLM agrees with the expectation value

$$
\langle\mathcal{O}(U)\rangle=\frac{1}{Z} \int d U \mathcal{O}(U) \operatorname{det} M[U] e^{-S_{g}[U]}
$$

defined in the path integral formalism.

The criterion for justification [24] is based on the magnitude of the drift term (2.6)

$$
v=\max _{x, \mu} \sqrt{\frac{1}{3} \operatorname{tr}\left(v_{x, \mu}^{\dagger} v_{x, \mu}\right)}
$$

If the histogram of this quantity falls off exponentially or faster, we can trust the results. This can be violated either by the excursion problem or by the singular drift problem as we mentioned in the Introduction. In the former case, it is the drift coming from the gauge action that shows slow fall-off in the histogram, while in the latter case, it is the drift coming from the fermion determinant.

The excursion problem can also be probed by the unitarity norm

$$
\mathcal{N}=\frac{1}{12 L_{\mathrm{t}} L_{\mathrm{s}}^{3}} \sum_{x, \mu} \operatorname{tr}\left(\mathcal{U}_{x, \mu}^{\dagger} \mathcal{U}_{x, \mu}-\mathbf{1}\right)
$$

which represents the distance of a configuration from the SU(3) manifold with $L_{\mathrm{t}}$ and $L_{\mathrm{s}}$ being the lattice size in the temporal and spatial directions, respectively. The unitarity norm (2.11) is positive semi-definite and it becomes zero if and only if all the link variables are unitary. Rapid growth of the unitarity norm typically signals the occurrence of the excursion problem. We note, however, that recent work [49] on $2 \mathrm{D} \mathrm{U}(1)$ theory with a $\theta$ term suggests that the CLM works even if the unitarity norm becomes large as far as the drift histogram falls off fast. ${ }^{1}$ Therefore, one cannot tell the validity of the CLM by looking at the unitarity norm alone.

\footnotetext{
${ }^{1}$ The fact that a large unitarity norm does not necessarily mean incorrect results has been noticed for a long time; See, for instance, ref. [50].
} 
In order to avoid the excursion problem, we use the gauge cooling [29, 30], which amounts to making a complexified gauge transformation

$$
\delta_{g} \mathcal{U}_{x, \mu}=g_{x} \mathcal{U}_{x, \mu} g_{x+\hat{\mu}}^{-1}, \quad g \in \mathrm{SL}(3, \mathrm{C})
$$

in such a way that the unitarity norm is minimized. Adding this procedure after each step of the Langevin-time evolution does not spoil the argument for justification as is shown in refs. $[23,24]$. The gauge cooling keeps all the link variables as close to unitary matrices as possible during the Langevin-time evolution.

As for physical observables, we calculate the Polyakov loop, the quark number and the chiral condensate. The Polyakov loop is given by

$$
P=\frac{1}{3 L_{\mathrm{s}}^{3}} \sum_{\vec{x}} \operatorname{tr}\left(\prod_{x_{4}=1}^{L_{\mathrm{t}}} U_{\left(\vec{x}, x_{4}\right), 4}\right),
$$

with $\vec{x}=\left(x_{1}, x_{2}, x_{3}\right)$ being a spatial coordinate on the lattice. The quark number $N_{\mathrm{q}}$ and the chiral condensate $\Sigma$ are defined by

$$
\begin{aligned}
N_{\mathrm{q}} & =\frac{1}{L_{\mathrm{t}}} \frac{\partial}{\partial \tilde{\mu}} \log Z=\frac{1}{L_{\mathrm{t}}}\left\langle\sum_{x} \frac{\eta_{4}(x)}{2} \operatorname{tr}\left(e^{\tilde{\mu}} M_{x+\hat{4}, x}^{-1} U_{x, 4}+e^{-\tilde{\mu}} M_{x-\hat{4}, x}^{-1} U_{x-\hat{4}, 4}^{-1}\right)\right\rangle, \\
\Sigma & =\frac{1}{L_{\mathrm{s}}^{3} L_{\mathrm{t}}} \frac{\partial}{\partial \tilde{m}} \log Z=\left\langle\operatorname{Tr} M^{-1}\right\rangle,
\end{aligned}
$$

where the latter trace $\operatorname{Tr}$ is taken not only for the color index but also for the spacetime index. These two quantities (2.14) and (2.15) are calculated by the so-called noisy estimator using 20 noise vectors.

\section{Results}

We have performed complex Langevin simulations at $\beta=5.7$ with degenerate quark mass $\tilde{m}=0.01$ on $8^{3} \times 16$ and $16^{3} \times 32$ lattices. We determine the lattice spacing as $a^{-1}=$ 4.65(1) GeV from the Sommer scale [51] by performing independent Hybrid Monte Carlo simulations on a $24^{3} \times 48$ lattice with $\tilde{\mu}=0$. The quark chemical potential is varied within the range $\tilde{\mu}=0.1-0.5$ on the $8^{3} \times 16$ lattice and $\tilde{\mu}=0.05-0.325$ on the $16^{3} \times 32$ lattice. The Langevin-time stepsize is chosen initially as $\varepsilon=10^{-4}$ and reduced adaptively [52] when the magnitude (2.10) of the drift becomes larger than the threshold 100. Measurement of the observables is made every $10^{-2}$ Langevin time. The total Langevin time after thermalization is $\tau=70-140$ for the $8^{3} \times 16$ lattice and $\tau=10-20$ for the $16^{3} \times 32$ lattice. The error bars of the physical observables are evaluated by the jackknife method with the bin sizes of 2 Langevin time on $8^{3} \times 16$ and 0.5 Langevin time on $16^{3} \times 32$.

\subsection{Validity of the CLM}

Let us first discuss the validity of the CLM in our simulation setup. In figure 1 we plot the distribution $p(v)$ for the magnitude of the drift term (2.10) coming from the gauge action (Left) and from the fermion determinant (Right), respectively, obtained by simulations on 

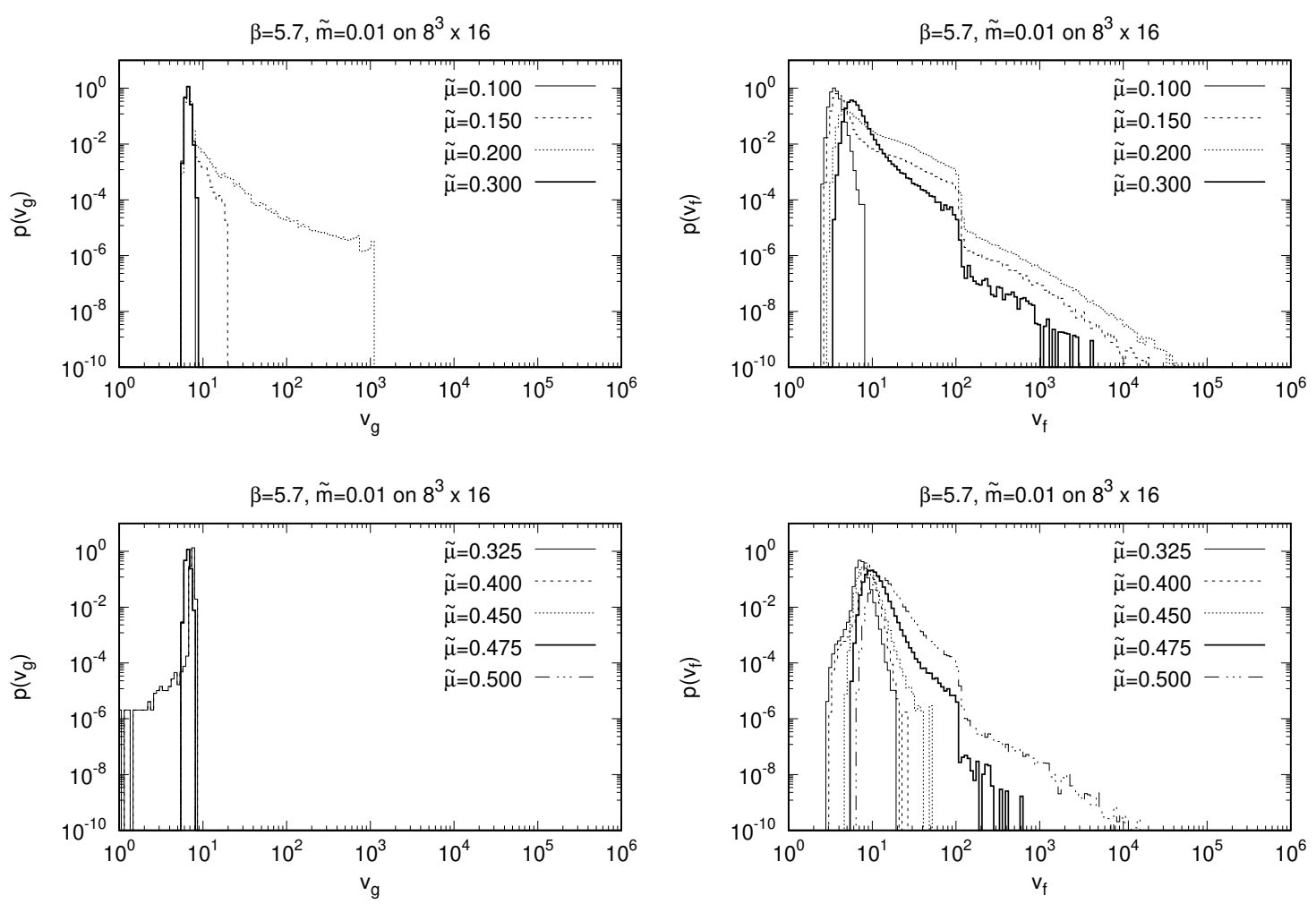

Figure 1. The probability distributions $p(v)$ of the drift term obtained from simulations on a $8^{3} \times 16$ lattice with $\beta=5.7$ and $\tilde{m}=0.01$ are plotted for the drift term $v_{\mathrm{g}}$ coming from the gauge action (Left) and $v_{\mathrm{f}}$ from the fermion determinant (Right). The upper and lower panels show the data points for $\tilde{\mu} \leq 0.3$ and $\tilde{\mu} \geq 0.325$, respectively.

a $8^{3} \times 16$ lattice with $\beta=5.7$ and $\tilde{m}=0.01$. For the sake of visibility, we separate the data points into two regions $\tilde{\mu} \leq 0.3$ and $\tilde{\mu} \geq 0.325$ and show them in the upper and lower panels, respectively. We find that the drift term coming from the gauge action shows slow fall-off for $\tilde{\mu}=0.2$, which implies that the excursion problem occurs only in this case. The drift term coming from the fermion determinant, on the other hand, shows slow fall-off for $0.15 \leq \tilde{\mu} \leq 0.3$ and $0.475 \leq \tilde{\mu}$ indicating the occurrence of the singular drift problem in these cases. ${ }^{2}$ Thus we conclude that the CLM gives correct results in the regions $\tilde{\mu} \simeq 0.1$ and $0.325 \leq \tilde{\mu} \leq 0.45$ on the $8^{3} \times 16$ lattice.

Figure 2 shows similar plots for a $16^{3} \times 32$ lattice with the same $\beta=5.7$ and $\tilde{m}=0.01$. Here we find that the excursion problem does not occur for any values of $\tilde{\mu}$, whereas the singular drift problem occurs for $\tilde{\mu}=0.325$. Thus we find that the CLM is expected to give correct results for $0.05 \leq \tilde{\mu} \leq 0.3$ on the $16^{3} \times 32$ lattice.

In figure 3 , we plot the Langevin-time histories of the unitarity norm (2.11). For the $8^{3} \times 16$ lattice (Left), we find that the history for $\tilde{\mu}=0.2$ looks quite violent, which is consistent with what we observe in figure 1 (Top-Left). For the $16^{3} \times 32$ lattice (Right),

\footnotetext{
${ }^{2}$ The sudden drop of the histogram around $v_{\mathrm{f}}=100$, which is also seen in figure 2 (Right), is an artifact of the chosen threshold for the adaptive stepsize mentioned at the beginning of section 3 .
} 

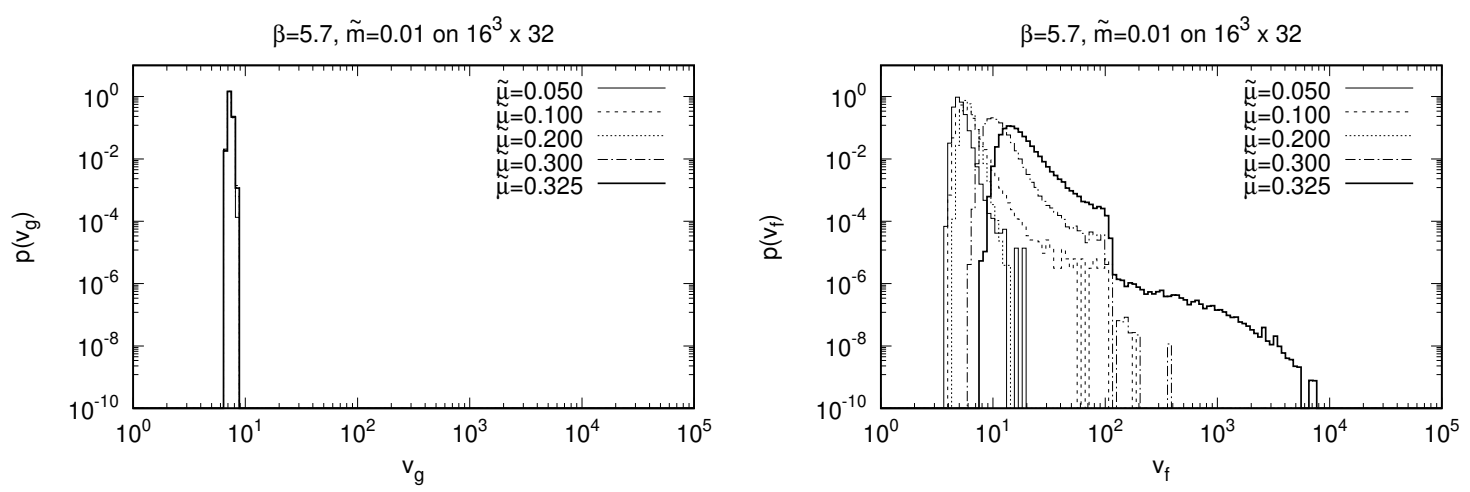

Figure 2. The probability distributions $p(v)$ of the drift term obtained from simulations on a $16^{3} \times 32$ lattice with $\beta=5.7$ and $\tilde{m}=0.01$ are plotted for the drift term $v_{\mathrm{g}}$ coming from the gauge action (Left) and $v_{\mathrm{f}}$ from the fermion determinant (Right).
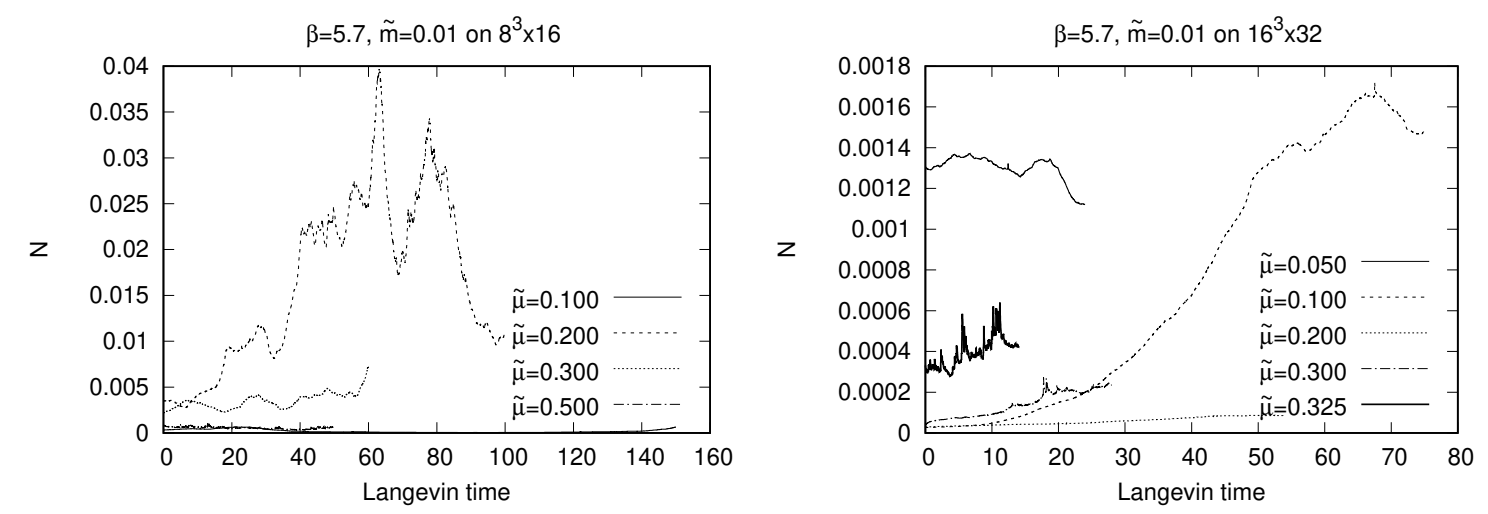

Figure 3. The Langevin-time histories of the unitarity norm (starting from the initial configuration) are plotted for $\beta=5.7$ and $\tilde{m}=0.01$ on the $8^{3} \times 16$ lattice (Left) and the $16^{3} \times 32$ lattice (Right).

on the other hand, the unitarity norm seems to be well under control; Note that the scale of the vertical axis here is an order of magnitude smaller than that in the Left panel. This is also consistent with what we observe in figure 2 (Left). Let us emphasize, however, that from the histories of the unitarity norm alone, we cannot judge the validity of the CLM unambiguously. Note also that the unitarity norm has a long autocorrelation time as one can see from figure 3. Fortunately, we find that the physical observables we investigate are not correlated with the unitarity norm, and they have a much shorter autocorrelation time. This is important because it allows us to calculate their expectation values reliably within a reasonable length of the total Langevin time.

\subsection{Physical observables}

In what follows, we present only the data points in the parameter region in which the criterion for justification is satisfied. In figure 4 (Top) we plot the real part of the Polyakov loop (2.13) against $\tilde{\mu}$ for $\beta=5.7$ and $\tilde{m}=0.01$ on the $8^{3} \times 16$ and the $16^{3} \times 32$ lattices. The results for the $8^{3} \times 16$ lattice are slightly nonzero and the results for the $16^{3} \times 32$ 
lattice are consistent with zero. Let us recall here that the Polyakov loop is an order parameter for the deconfining transition. The interpretation of our results requires some care, though. Note that the spatial size of our lattice is $a L_{\mathrm{s}}=0.36 \mathrm{fm}$ and $0.68 \mathrm{fm}$ for $L_{\mathrm{s}}=8$ and 16, respectively, which are smaller than the typical length scale of QCD, namely $\Lambda_{\mathrm{LQCD}}^{-1} \sim 1 \mathrm{fm}$. Thus the situation we are simulating should be regarded as QCD in a small box, where the notion of quark confinement does not make sense. In fact, the temperature is $T \sim 290 \mathrm{MeV}$ and $145 \mathrm{MeV}$ for $L_{\mathrm{t}}=16$ and 32 , respectively, which are higher than or close to the critical temperature $T_{\mathrm{c}} \sim 170 \mathrm{MeV}$ for the deconfining transition in QCD with four-flavor staggered quarks [53] with $m / T=0.2$, where large physical volume is implicitly assumed. The Polyakov loop being either small or zero in our setup simply confirms that we are probing the "low temperature" behavior of such a finite size system due to the chosen aspect ratio of our lattice.

In figure 4 (Middle) we plot the quark number (2.14) against $\tilde{\mu}$ for $\beta=5.7$ and $\tilde{m}=0.01$ on the $8^{3} \times 16$ and $16^{3} \times 32$ lattices. On both lattices, we observe a plateau at the height of $N_{\mathrm{q}}=24$. In order to understand this behavior, let us recall that the physical extent of our lattice is too small to create a baryon in it. The effective gauge coupling is small due to the asymptotic freedom, which makes the Fermi distribution of quarks qualitatively valid. At sufficiently large $\tilde{\mu}$, the path integral is therefore dominated by a state obtained from the vacuum by creating quarks with momentum $\vec{p}$ satisfying $\sqrt{\vec{p}^{2}+m_{\text {eff }}^{2}} \leq \tilde{\mu}$, where $m_{\text {eff }}$ is the effective mass including quantum corrections. ${ }^{3}$ It should be noted here that the momentum is discretized in a finite box as $\vec{p}=\left(2 \pi / L_{\mathrm{s}}\right) \vec{n}$ with $\vec{n}$ being a 3D integer vector. In particular, for $m_{\mathrm{eff}} \leq \tilde{\mu} \leq \mu_{1}$, where $\mu_{1}=\sqrt{\left(2 \pi / L_{\mathrm{S}}\right)^{2}+m_{\mathrm{eff}}^{2}}$, only the quarks with $\vec{p}=0$ are created. The height of the plateau is therefore given by the internal degrees of freedom $N_{\mathrm{f}} \times N_{\mathrm{c}} \times N_{\text {spin }}=4 \times 3 \times 2=24$, where $N_{\mathrm{f}}, N_{\mathrm{c}}$ and $N_{\text {spin }}$ are the number of flavors, the number of colors and the number of spin degrees of freedom, respectively. ${ }^{4}$ In figure 4 (Middle), we also observe that our data start to leave the plateau for larger $\tilde{\mu}$, which can be understood as the effects of quarks with the first non-zero momenta being created. The value of $\tilde{\mu}$ at which this growth of $N_{\mathrm{q}}$ occurs is smaller than $\mu_{1}$ defined above, which can be understood as a result of finite temperature effects. ${ }^{5}$ Note that $\mu_{1}$ becomes smaller as the lattice becomes larger, which is clearly reflected in our results.

The appearance of such plateaus in the quark number for QCD in a finite box was discussed in the case of free theory using the naive lattice action for fermions [55]. See also ref. [56] for such behaviors based on one-loop perturbative calculations in the continuum QCD on a small $\mathrm{S}^{3}$. In a separate paper [57], we will report on our results of the CLM for larger $\beta$, which are compared with perturbative results obtained with staggered fermions. This plateau behavior should not be confused with the quark number saturation that occurs at much larger $\tilde{\mu}$. In that case, the path integral is dominated by a state with all the sites

\footnotetext{
${ }^{3}$ Here we neglect finite lattice spacing effects assuming that we are close to the continuum limit.

${ }^{4}$ In the case of two-flavor Wilson fermions, the height of the plateau is found to be 12, which agrees with $N_{\mathrm{f}} \times N_{\mathrm{c}} \times N_{\text {spin }}=2 \times 3 \times 2=12$. We thank Manuel Scherzer for confirming this with his data [54].

${ }^{5}$ For instance, for the $16^{3} \times 32$ lattice, a sharp transition to the second plateau with the height $N_{\mathrm{q}}=$ $24 \times(1+6)=168$ is expected to occur at $\mu_{1} \sim 0.4$ using $m_{\text {eff }}^{2} \sim 0.1$ if the temperature were zero. However, our results show an increase of $N_{\mathrm{q}}$ already at $\tilde{\mu} \sim 0.3$ due to finite temperature effects.
} 

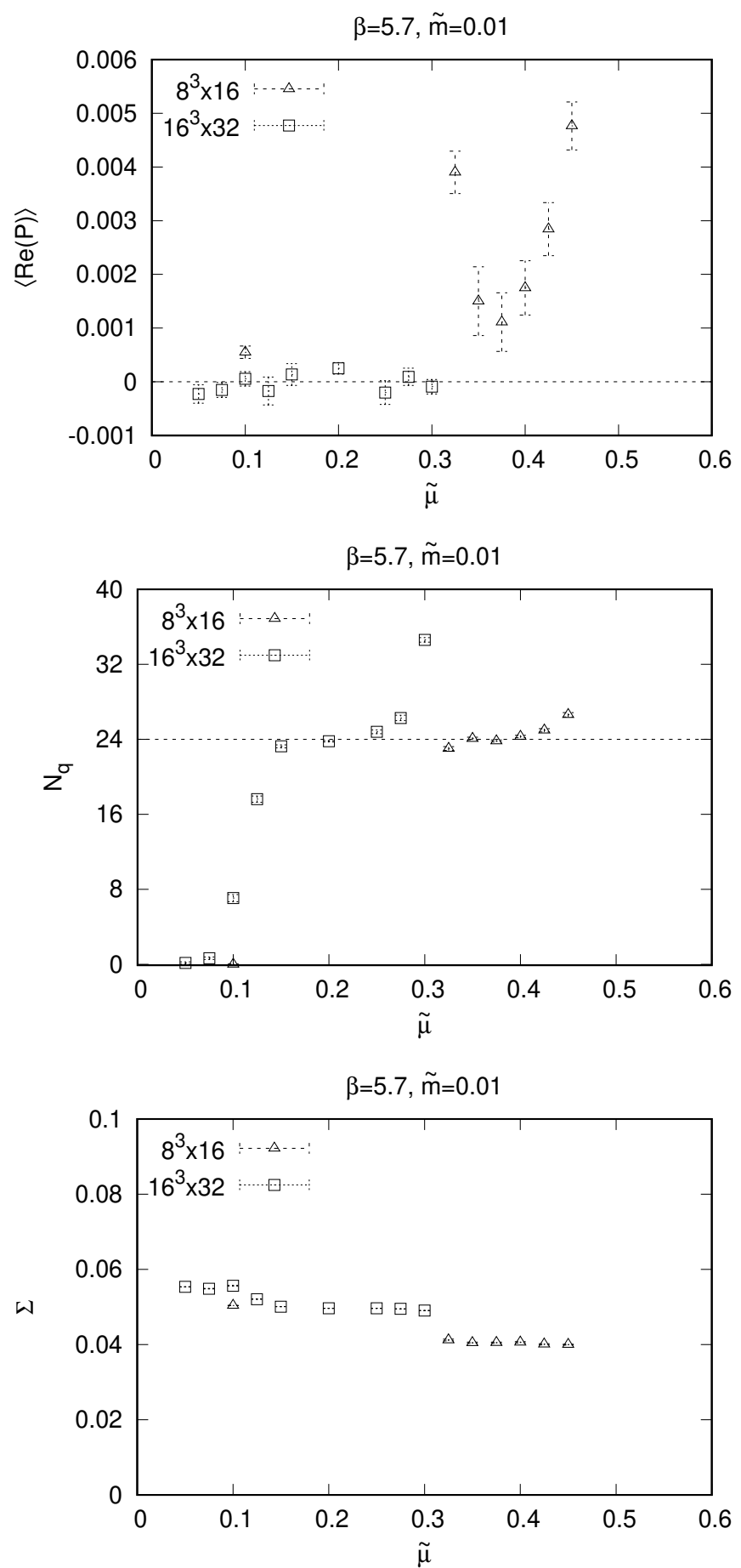

Figure 4. The real part of the Polyakov loop $P$ (Top), the quark number $N_{\mathrm{q}}$ (Middle) and the chiral condensate $\Sigma$ (Bottom) are plotted against the quark chemical potential $\tilde{\mu}$ for $\beta=5.7$ and $\tilde{m}=0.01$ on the $8^{3} \times 16$ and $16^{3} \times 32$ lattices. 
being occupied by fermions. Taking the internal degrees of freedom into account, the height of the plateau becomes $N_{\mathrm{f}} \times N_{\mathrm{c}} \times N_{\text {spin }} \times L_{\mathrm{s}}^{3}=24 \times L_{\mathrm{s}}^{3}$, which is much higher than 24 .

In figure 4 (Bottom) we show our results for the chiral condensate (2.15). The plateau behaviors appear here as well because of the "low temperature", where changing $\tilde{\mu}$ a little cannot create quarks at higher energy levels. The plateau corresponding to the state with the zero-momentum quarks appears with the height only slightly lower than that corresponding to the state that dominates at $\tilde{\mu}=0$. This suggests that the chiral symmetry for $\tilde{m}=0$, which is considered to be spontaneously broken at $\tilde{\mu}=0$, does not get restored by increasing $\tilde{\mu}$ in the present parameter regime.

The plateau behaviors observed above in the quark number and the chiral condensate are analogous to those in two-color $\mathrm{QCD}^{6}$ using two-flavor Wilson fermions on a $3^{3} \times 64$ lattice at $\beta=24$ with finite $\mu$ [58]. In that case, however, the height of the plateau in the quark number does not agree with the free fermion results, which is in contrast to our results for the $\mathrm{SU}(3)$ gauge group on $8^{3} \times 16$ and $16^{3} \times 32$ lattices.

\section{Summary and discussions}

In this paper we have applied the CLM to QCD at finite density with the plaquette gauge action and the four-flavor staggered fermions on $8^{3} \times 16$ and $16^{3} \times 32$ lattices. While the spatial size of our lattice is still as small as $a L_{\mathrm{s}}=0.36 \mathrm{fm}$ and $0.68 \mathrm{fm}$ for $L_{\mathrm{s}}=8$ and 16 , we find that the criterion for correct convergence is satisfied for $\mu=1.5-2.1 \mathrm{GeV}$ on the $8^{3} \times 16$ lattice and for $\mu=0.23-1.4 \mathrm{GeV}$ on the $16^{3} \times 32$ lattice with $T \sim 290 \mathrm{MeV}$ and $145 \mathrm{MeV}$, respectively. These parameter regimes cannot be reached by conventional methods, such as the density of states method and the Taylor expansion method. Thus our results clearly demonstrate a big advantage of the CLM in overcoming the sign problem in finite density QCD.

Let us also mention that the previous work [11] shows that the CLM works on a $4^{3} \times 8$ lattice with the same $\beta$ but only with the aid of the deformation technique [59], which is actually not needed for the lattice size in the present work. Thus we find that the situation becomes better for a larger lattice, which is also seen by comparing our results for $8^{3} \times 16$ and $16^{3} \times 32$ lattices in section 3.1 .

One of our main physical results is that the quark number exhibits a plateau behavior as a function of the quark chemical potential with the height of 24 at sufficiently large $\mu$. This has been interpreted as the creation of quarks with zero momentum, which has the internal degrees of freedom $N_{\mathrm{f}} \times N_{\mathrm{c}} \times N_{\text {spin }}=4 \times 3 \times 2=24$. We may regard it as the first step towards the formation of the Fermi surface, which plays a crucial role in color superconductivity.

It is of particular importance to perform similar calculations with larger lattices. That will enable us to observe the growth of the Fermi sphere with moderate values of $\mu$ thanks to better momentum resolution. Note that color superconductivity is expected to occur due to Cooper pairing of quarks near the Fermi surface, which is possible even at weak

\footnotetext{
${ }^{6}$ This model has no sign problem even at finite density and hence the standard hybrid Monte Carlo algorithm is applicable.
} 
coupling or in a small physical volume [60]. For this reason, we are currently exploring the larger $\beta$ regime, where we can compare our results against perturbative calculations [57]. In particular, we are trying to observe a departure from perturbative behaviors as $\beta$ gets smaller than some critical value, which would signal the onset of color superconductivity.

\section{Acknowledgments}

We thank E. Itou, A. Ohnishi and M. Scherzer for important discussions, which enabled us to establish the interpretation of the plateau behavior observed in our results for the quark number. The authors are also grateful to M.P. Lombardo for bringing our attention to ref. [55] and to Y. Asano, T. Kaneko and T. Yokota for collaborations in the ongoing projects related to the present work. This research was supported by MEXT as "Priority Issue on Post-K computer" (Elucidation of the Fundamental Laws and Evolution of the Universe) and as "Program for Promoting Researches on the Supercomputer Fugaku" (Simulation for basic science: from fundamental laws of particles to creation of nuclei). It is also supported by Joint Institute for Computational Fundamental Science (JICFuS). Computations were carried out using computational resources of the $\mathrm{K}$ computer provided by the RIKEN Center for Computational Science through the HPCI System Research project (Project ID:hp180178, hp190159) and the Oakbridge-CX provided by the Information Technology Center at the University of Tokyo through the HPCI System Research project (Project ID:hp200079). Y.N. and J.N. were supported in part by JSPS KAKENHI Grant Numbers JP16H03988, JP18K03638. S.S. was supported by the MEXT-Supported Program for the Strategic Research Foundation at Private Universities "Topological Science" (Grant No. S1511006). S.T. was supported by the RIKEN Special Postdoctoral Researchers Program.

Open Access. This article is distributed under the terms of the Creative Commons Attribution License (CC-BY 4.0), which permits any use, distribution and reproduction in any medium, provided the original author(s) and source are credited.

\section{References}

[1] G. Parisi, On complex probabilities, Phys. Lett. B 131 (1983) 393 [InSPIRE].

[2] J.R. Klauder, Coherent state Langevin equations for canonical quantum systems with applications to the quantized Hall effect, Phys. Rev. A 29 (1984) 2036 [INSPIRE].

[3] C.E. Berger, L. Rammelmüller, A.C. Loheac, F. Ehmann, J. Braun and J.E. Drut, Complex Langevin and other approaches to the sign problem in quantum many-body physics, arXiv: 1907.10183 [INSPIRE].

[4] D. Sexty, Simulating full QCD at nonzero density using the complex Langevin equation, Phys. Lett. B $\mathbf{7 2 9}$ (2014) 108 [arXiv:1307.7748] [InSPIRE].

[5] G. Aarts, E. Seiler, D. Sexty and I.-O. Stamatescu, Simulating QCD at nonzero baryon density to all orders in the hopping parameter expansion, Phys. Rev. D 90 (2014) 114505 [arXiv:1408.3770] [INSPIRE]. 
[6] Z. Fodor, S.D. Katz, D. Sexty and C. Török, Complex Langevin dynamics for dynamical QCD at nonzero chemical potential: A comparison with multiparameter reweighting, Phys. Rev. D 92 (2015) 094516 [arXiv: 1508.05260] [InSPIRE].

[7] D.K. Sinclair and J.B. Kogut, Exploring complex-Langevin methods for finite-density QCD, PoS LATTICE2015 (2016) 153 [arXiv: 1510.06367] [INSPIRE].

[8] D.K. Sinclair and J.B. Kogut, Complex Langevin for lattice $Q C D$ at $T=0$ and $\mu \geq 0, P o S$ LATTICE2016 (2016) 026 [arXiv:1611.02312] [INSPIRE].

[9] D.K. Sinclair and J.B. Kogut, Complex Langevin simulations of QCD at finite density Progress report, EPJ Web Conf. 175 (2018) 07031 [arXiv:1710.08465] [INSPIRE].

[10] D.K. Sinclair and J.B. Kogut, Complex Langevin for lattice $Q C D$, PoS LATTICE2018 (2018) 143 [arXiv : 1810.11880] [INSPIRE].

[11] K. Nagata, J. Nishimura and S. Shimasaki, Complex Langevin calculations in finite density QCD at large $\mu / T$ with the deformation technique, Phys. Rev. D 98 (2018) 114513 [arXiv: 1805.03964] [INSPIRE].

[12] Y. Ito, H. Matsufuru, J. Nishimura, S. Shimasaki, A. Tsuchiya and S. Tsutsui, Exploring the phase diagram of finite density $Q C D$ at low temperature by the complex Langevin method, PoS LATTICE2018 (2018) 146 [arXiv:1811.12688] [INSPIRE].

[13] S. Tsutsui, Y. Ito, H. Matsufuru, J. Nishimura, S. Shimasaki and A. Tsuchiya, Can the complex Langevin method see the deconfinement phase transition in $Q C D$ at finite density?, PoS LATTICE2018 (2018) 144 [arXiv:1811.07647] [INSPIRE].

[14] S. Tsutsui, Y. Ito, H. Matsufuru, J. Nishimura, S. Shimasaki and A. Tsuchiya, Exploring the finite density QCD based on the complex Langevin method, JPS Conf. Proc. 26 (2019) 024012 [INSPIRE].

[15] J.B. Kogut and D.K. Sinclair, Applying complex Langevin simulations to lattice QCD at finite density, Phys. Rev. D 100 (2019) 054512 [arXiv:1903.02622] [INSPIRE].

[16] D. Sexty, Calculating the equation of state of dense quark-gluon plasma using the complex Langevin equation, Phys. Rev. D 100 (2019) 074503 [arXiv:1907.08712] [INSPIRE].

[17] D.K. Sinclair and J.B. Kogut, Applying complex Langevin to lattice $Q C D$ at finite $\mu, P o S$ LATTICE2019 (2019) 245 [arXiv:1910.11412] [INSPIRE].

[18] S. Tsutsui, Y. Ito, H. Matsufuru, J. Nishimura, S. Shimasaki and A. Tsuchiya, Exploring the QCD phase diagram at finite density by the complex Langevin method on a $16^{3} \times 32$ lattice, PoS LATTICE2019 (2019) 151 [arXiv:1912.00361] [INSPIRE].

[19] M. Scherzer, D. Sexty and I.-O. Stamatescu, Deconfinement transition line with the complex Langevin equation up to $\mu / T \sim 5$, Phys. Rev. D 102 (2020) 014515 [arXiv:2004.05372] [INSPIRE].

[20] G. Aarts, E. Seiler and I.-O. Stamatescu, The complex Langevin method: When can it be trusted?, Phys. Rev. D 81 (2010) 054508 [arXiv:0912.3360] [INSPIRE].

[21] G. Aarts, F.A. James, E. Seiler and I.-O. Stamatescu, Complex Langevin: Etiology and diagnostics of its main problem, Eur. Phys. J. C 71 (2011) 1756 [arXiv:1101.3270] [INSPIRE].

[22] J. Nishimura and S. Shimasaki, New insights into the problem with a singular drift term in the complex Langevin method, Phys. Rev. D 92 (2015) 011501 [arXiv:1504.08359] [INSPIRE]. 
[23] K. Nagata, J. Nishimura and S. Shimasaki, Justification of the complex Langevin method with the gauge cooling procedure, PTEP 2016 (2016) 013B01 [arXiv: 1508.02377] [INSPIRE].

[24] K. Nagata, J. Nishimura and S. Shimasaki, Argument for justification of the complex Langevin method and the condition for correct convergence, Phys. Rev. D 94 (2016) 114515 [arXiv: 1606.07627] [INSPIRE].

[25] L.L. Salcedo, Does the complex Langevin method give unbiased results?, Phys. Rev. D 94 (2016) 114505 [arXiv:1611.06390] [INSPIRE].

[26] G. Aarts, E. Seiler, D. Sexty and I.-O. Stamatescu, Complex Langevin dynamics and zeroes of the fermion determinant, JHEP 05 (2017) 044 [Erratum ibid. 01 (2018) 128] [arXiv: 1701.02322] [INSPIRE].

[27] K. Nagata, J. Nishimura and S. Shimasaki, Testing the criterion for correct convergence in the complex Langevin method, JHEP 05 (2018) 004 [arXiv:1802.01876] [INSPIRE].

[28] A. Mollgaard and K. Splittorff, Complex Langevin Dynamics for chiral Random Matrix Theory, Phys. Rev. D 88 (2013) 116007 [arXiv:1309.4335] [INSPIRE].

[29] E. Seiler, D. Sexty and I.-O. Stamatescu, Gauge cooling in complex Langevin for QCD with heavy quarks, Phys. Lett. B $\mathbf{7 2 3}$ (2013) 213 [arXiv:1211.3709] [INSPIRE].

[30] G. Aarts, L. Bongiovanni, E. Seiler, D. Sexty and I.-O. Stamatescu, Controlling complex Langevin dynamics at finite density, Eur. Phys. J. A 49 (2013) 89 [arXiv:1303.6425] [INSPIRE].

[31] M. Scherzer, E. Seiler, D. Sexty and I.-O. Stamatescu, Complex Langevin and boundary terms, Phys. Rev. D 99 (2019) 014512 [arXiv:1808.05187] [InSPIRE].

[32] M. Scherzer, E. Seiler, D. Sexty and I.-O. Stamatescu, Controlling complex Langevin simulations of lattice models by boundary term analysis, Phys. Rev. D 101 (2020) 014501 [arXiv: 1910.09427] [INSPIRE].

[33] M. Fukugita, H. Mino, M. Okawa and A. Ukawa, Finite size test for the finite temperature chiral phase transition in lattice QCD, Phys. Rev. Lett. 65 (1990) 816 [INSPIRE].

[34] Z. Fodor and S.D. Katz, A new method to study lattice $Q C D$ at finite temperature and chemical potential, Phys. Lett. B 534 (2002) 87 [hep-lat/0104001] [INSPIRE].

[35] M. D'Elia and M.-P. Lombardo, Finite density QCD via imaginary chemical potential, Phys. Rev. D 67 (2003) 014505 [hep-lat/0209146] [INSPIRE].

[36] M. D'Elia and M.P. Lombardo, QCD thermodynamics from an imaginary mu(B): Results on the four flavor lattice model, Phys. Rev. D 70 (2004) 074509 [hep-lat/0406012] [INSPIRE].

[37] V. Azcoiti, G. Di Carlo, A. Galante and V. Laliena, Finite density QCD: A new approach, JHEP 12 (2004) 010 [hep-lat/0409157] [INSPIRE].

[38] V. Azcoiti, G. Di Carlo, A. Galante and V. Laliena, Phase diagram of QCD with four quark flavors at finite temperature and baryon density, Nucl. Phys. B 723 (2005) 77 [hep-lat/0503010] [INSPIRE].

[39] Z. Fodor, S.D. Katz and C. Schmidt, The density of states method at non-zero chemical potential, JHEP 03 (2007) 121 [hep-lat/0701022] [INSPIRE].

[40] M. D'Elia, F. Di Renzo and M.P. Lombardo, The strongly interacting quark gluon plasma, and the critical behaviour of QCD at imaginary mu, Phys. Rev. D 76 (2007) 114509 [arXiv:0705.3814] [INSPIRE]. 
[41] G. Endrodi, Z. Fodor, S.D. Katz, D. Sexty, K.K. Szabo and C. Török, Applying constrained simulations for low temperature lattice QCD at finite baryon chemical potential, Phys. Rev. D 98 (2018) 074508 [arXiv: 1807.08326] [INSPIRE].

[42] H.-S. Chen and X.-Q. Luo, Phase diagram of QCD at finite temperature and chemical potential from lattice simulations with dynamical Wilson quarks, Phys. Rev. D 72 (2005) 034504 [hep-lat/0411023] [INSPIRE].

[43] P. de Forcrand and S. Kratochvila, Finite density QCD with a canonical approach, Nucl. Phys. B Proc. Suppl. 153 (2006) 62 [hep-lat/0602024] [InSPIRE].

[44] A. Li, A. Alexandru, K.-F. Liu and X. Meng, Finite density phase transition of QCD with $N_{f}=4$ and $N_{f}=2$ using canonical ensemble method, Phys. Rev. D 82 (2010) 054502 [arXiv: 1005.4158] [INSPIRE].

[45] S. Takeda, Y. Kuramashi and A. Ukawa, On the phase of quark determinant in lattice QCD with finite chemical potential, Phys. Rev. D 85 (2012) 096008 [arXiv:1111.6363] [InSPIRE].

[46] X.-Y. Jin, Y. Kuramashi, Y. Nakamura, S. Takeda and A. Ukawa, Finite size scaling study of $N_{f}=4$ finite density QCD on the lattice, Phys. Rev. D 88 (2013) 094508 [arXiv:1307.7205] [INSPIRE].

[47] O. Philipsen, Constraining the QCD phase diagram at finite temperature and density, PoS LATTICE2019 (2019) 273 [arXiv:1912.04827] [INSPIRE].

[48] A. Ohnishi, Approaches to QCD phase diagram; effective models, strong-coupling lattice QCD, and compact stars, J. Phys. Conf. Ser. 668 (2016) 012004 [arXiv:1512.08468] [INSPIRE].

[49] M. Hirasawa, A. Matsumoto, J. Nishimura and A. Yosprakob, Complex Langevin analysis of $2 D \mathrm{U}(1)$ gauge theory on a torus with a $\theta$ term, JHEP 09 (2020) 023 [arXiv:2004.13982] [INSPIRE].

[50] J. Berges and D. Sexty, Real-time gauge theory simulations from stochastic quantization with optimized updating, Nucl. Phys. B 799 (2008) 306 [arXiv:0708.0779] [InSPIRE].

[51] R. Sommer, A new way to set the energy scale in lattice gauge theories and its applications to the static force and alpha-s in SU(2) Yang-Mills theory, Nucl. Phys. B 411 (1994) 839 [hep-lat/9310022] [INSPIRE].

[52] G. Aarts, F.A. James, E. Seiler and I.-O. Stamatescu, Adaptive stepsize and instabilities in complex Langevin dynamics, Phys. Lett. B 687 (2010) 154 [arXiv:0912.0617] [INSPIRE].

[53] J. Engels, R. Joswig, F. Karsch, E. Laermann, M. Lutgemeier and B. Petersson, Thermodynamics of four flavor QCD with improved staggered fermions, Phys. Lett. B 396 (1997) 210 [hep-lat/9612018] [INSPIRE].

[54] M. Scherzer, Phase transitions in lattice gauge theories: From the numerical sign problem to machine learning. Ph.D. Thesis, Heidelberg University, Germany (2019), [InSPIRE].

[55] H. Matsuoka and M. Stone, Thermal distribution functions and finite size effects for lattice fermions, Phys. Lett. B 136 (1984) 204 [INSPIRE].

[56] S. Hands, T.J. Hollowood and J.C. Myers, QCD with chemical potential in a small hyperspherical box, JHEP 07 (2010) 086 [arXiv: 1003.5813] [INSPIRE].

[57] T. Yokota et al., work in progress. 
[58] S. Hands, T.J. Hollowood and J.C. Myers, Numerical study of the two color attoworld, JHEP 12 (2010) 057 [arXiv: 1010.0790] [INSPIRE].

[59] Y. Ito and J. Nishimura, The complex Langevin analysis of spontaneous symmetry breaking induced by complex fermion determinant, JHEP 12 (2016) 009 [arXiv:1609.04501] [INSPIRE].

[60] P. Amore, M.C. Birse, J.A. McGovern and N.R. Walet, Color superconductivity in finite systems, Phys. Rev. D 65 (2002) 074005 [hep-ph/0110267] [INSPIRE]. 